\title{
The Nature and Role of Bridged Carbonyl Intermediates in the Ultrafast Photo-induced Rearrangement of $\mathrm{Ru}_{3}(\mathrm{CO})_{12}$
}

Elizabeth A. Glascoe, Matthias F. Kling, ${ }^{\neq}$Jennifer E. Shanoski, and Charles B. Harris* Contribution from the Department of Chemistry, University of California, Berkeley, California 94720, and Chemical Sciences Division, Lawrence Berkeley National Laboratory, Berkeley, California 94720

*E-mail: cbharris@berkeley.edu

* Present address: FOM Institute for Atomic and Molecular Physics (AMOLF), Kruislaan 407, 1098 SJ Amsterdam, The Netherlands. 


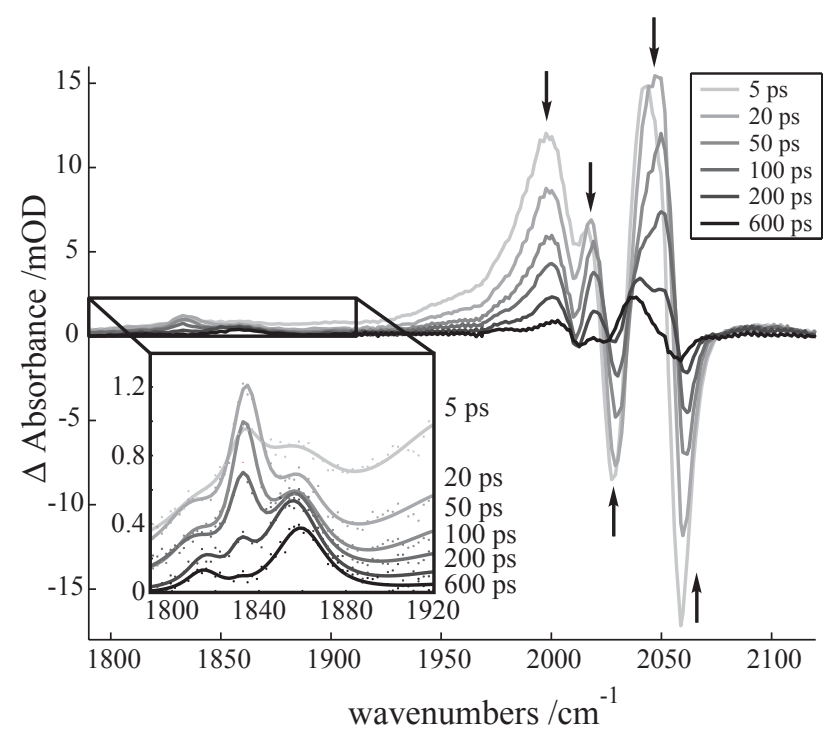

Figure S1. $\mathrm{Ru}_{3}(\mathrm{CO})_{12}$ in Cyclohexane excited with $266 \mathrm{~nm}$ light $(0.3 \mu \mathrm{J})$. Arrows indicate changes with increasing delay time.

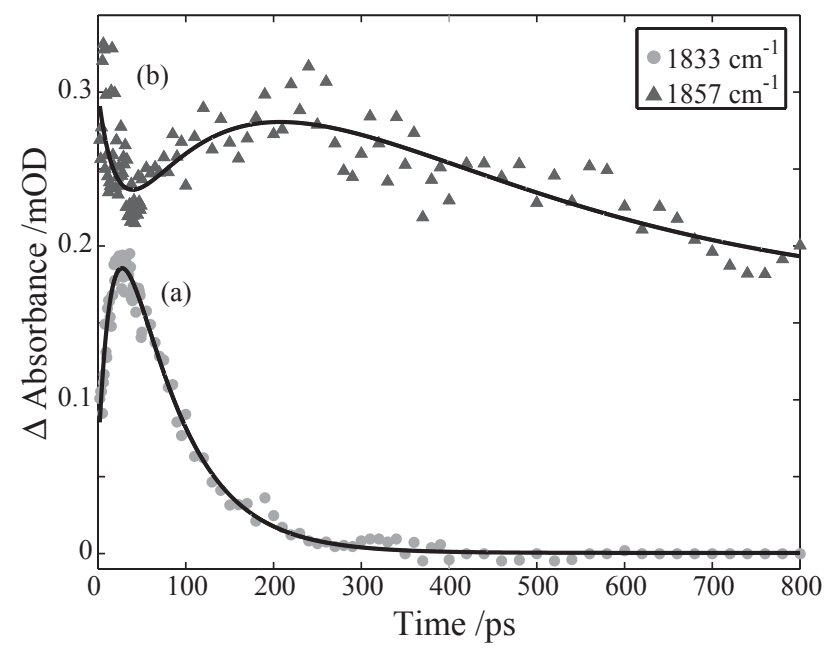

Figure S2. Kinetics of $\mathrm{Ru}_{3}(\mathrm{CO})_{12}$ in cyclohexane excited with $266 \mathrm{~nm}$ : (a) $1833 \mathrm{~cm}^{-1}$ peak areas (dots) together with a bi-exponential fit, (b) $1857 \mathrm{~cm}^{-1}$ peak areas (triangles) together with the corresponding fit; the data at $1857 \mathrm{~cm}^{-1}$ was raised vertically to allow an easy visualization of the data taken at 1833 $\mathrm{cm}^{-1}$. 


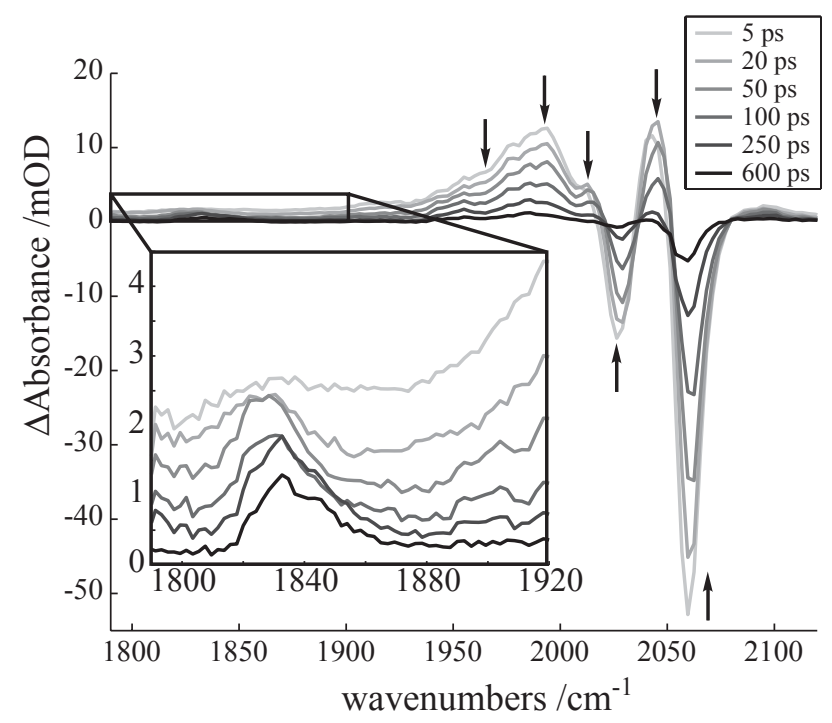

Figure S3. Difference spectra of $\mathrm{Ru}_{3}(\mathrm{CO})_{12}$ excited with $266 \mathrm{~nm}(0.3 \mu \mathrm{J})$ in a neat solution of THF. Arrows indicate changes with increasing delay time. 


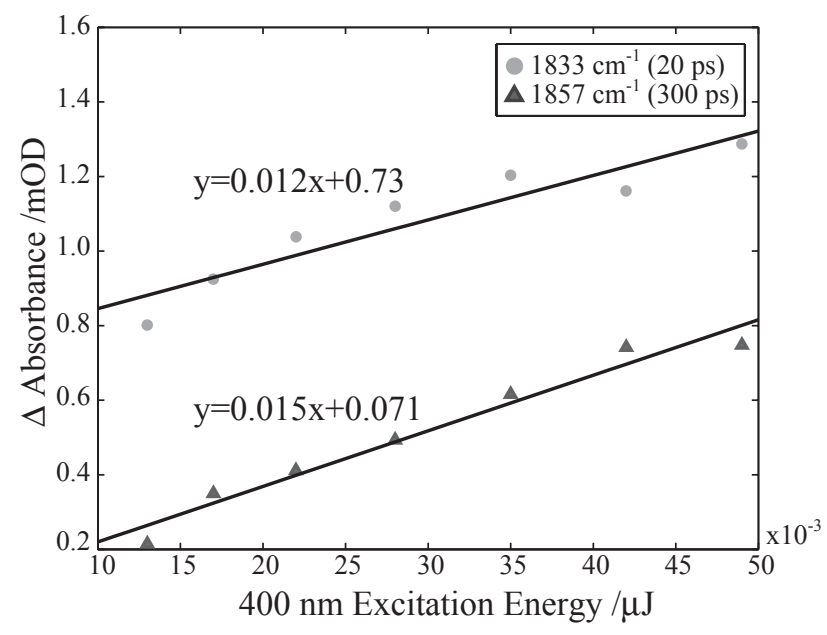

Figure S4. Power dependent peak amplitudes after photo-exciting $\mathrm{Ru}_{3}(\mathrm{CO})_{12}$ in cyclohexane with various energies of 400-nm light. The linear dependence of peak amplitude on excitation energy indicates that both peaks result from single photon processes. The energy was measured after a 400/800 $\mathrm{nm}$ beam splitter in order to maximize measurement accuracy, subsequently the beam passes through a series of optics prior to impinging on the sample. Therefore, the energy measurements reflect relative pump energies and not the actual energies of the 400-nm pulses used to photo-excite the sample. Because the peaks appear at different times it was necessary to measure the $1833 \mathrm{~cm}^{-1}$ peak at 20 ps and the $1857 \mathrm{~cm}^{-1}$ peak at $300 \mathrm{ps}$. 
Table S1. Geometry optimized bond lengths and angles for the parent molecule $\mathrm{Ru}_{3}(\mathrm{CO})_{12}$ prior to excitation.

\begin{tabular}{|c|c|c|c|}
\hline Distance $(\AA)$ & $\begin{array}{l}\text { Basis } \\
\text { Set } \mathbf{A}^{a} \\
\end{array}$ & $\begin{array}{l}\text { Basis } \\
\text { Set B }{ }^{a} \\
\end{array}$ & $\operatorname{exptl}^{b}$ \\
\hline $\mathrm{Ru}-\mathrm{Ru}$ & 2.924 & 2.929 & 2.854 \\
\hline $\mathrm{Ru}-\mathrm{C}_{\mathrm{ax}}$ & 1.969 & 1.971 & 1.942 \\
\hline $\mathrm{Ru}^{\cdots} \mathrm{O}_{\mathrm{ax}}$ & 3.115 & 3.116 & 3.071 \\
\hline $\mathrm{C}-\mathrm{O}_{\mathrm{ax}}$ & 1.151 & 1.151 & 1.133 \\
\hline $\mathrm{Ru}-\mathrm{C}_{\mathrm{eq}}$ & 1.944 & 1.943 & 1.921 \\
\hline $\mathrm{Ru} \ldots \mathrm{O}_{\mathrm{eq}}$ & 3.093 & 3.093 & 3.048 \\
\hline $\mathrm{C}-\mathrm{O}_{\mathrm{eq}}$ & 1.150 & 1.150 & 1.127 \\
\hline \multicolumn{4}{|l|}{ Angles } \\
\hline $\mathrm{Ru}-\mathrm{Ru}-\mathrm{Ru}$ & 60.00 & 60.00 & 60.00 \\
\hline $\mathrm{Ru}-\mathrm{Ru}-\mathrm{C}_{\mathrm{eq}}$ & 99.99 & 100.18 & 97.95 \\
\hline $\mathrm{C}_{\mathrm{eq}}-\mathrm{Ru}-\mathrm{C}_{\mathrm{eq}}$ & 101.63 & 101.48 & 104.09 \\
\hline $\begin{array}{l}\mathrm{Ru}-\mathrm{Ru}-\mathrm{C}_{\mathrm{ax}} \\
\text { (acute) }\end{array}$ & 78.98 & 78.53 & 88.99 \\
\hline $\begin{array}{l}\mathrm{Ru}-\mathrm{Ru}-\mathrm{C}_{\mathrm{ax}} \\
\text { (obtuse) }\end{array}$ & 95.29 & 95.91 & 90.08 \\
\hline $\mathrm{C}_{\mathrm{ax}}-\mathrm{Ru}-\mathrm{C}_{\mathrm{eq}}$ & 92.07 & 92.01 & 90.34 \\
\hline $\mathrm{C}_{\mathrm{ax}}-\mathrm{Ru}-\mathrm{C}_{\mathrm{ax}}$ & 173.45 & 173.65 & 178.33 \\
\hline $\mathrm{Ru}-\mathrm{C}-\mathrm{O}_{\mathrm{ax}}$ & 173.01 & 172.67 & 172.98 \\
\hline $\mathrm{Ru}-\mathrm{C}-\mathrm{O}_{\mathrm{eq}}$ & 177.63 & 177.64 & 178.91 \\
\hline
\end{tabular}

$\mathrm{C}_{\text {eq }}-\mathrm{Ru}-\mathrm{Ru}-\mathrm{C}_{\mathrm{eq}} \quad 24.11 \quad 25.74 \quad$ na

$\mathrm{C}_{\mathrm{ax}}-\mathrm{Ru}-\mathrm{Ru}-\mathrm{C}_{\mathrm{ax}} \quad 28.72 \quad 30.70 \quad$ na

\footnotetext{
${ }^{a}$ See theoretical methods section (IID) for basis set information. ${ }^{b}$ ref 5
} 
Table S2. Calculated vibrational frequencies $\left(\mathrm{cm}^{-1}\right)$ and relative amplitudes for the parent complex, $\mathrm{Ru}_{3}(\mathrm{CO})_{12}$, and the metal-metal cleavage complex, $\mathbf{I}$.

\begin{tabular}{c|c|c|c|c|c}
\hline \multicolumn{2}{c|}{ Ru $_{\mathbf{3}}(\mathbf{C O})_{\mathbf{1 2}}$} & \multicolumn{4}{c}{ Complex I } \\
\hline $\begin{array}{c}\text { Basis Set } A \\
\text { Unsolv. }\end{array}$ & $\begin{array}{c}\text { Basis Set } B \\
\text { Unsolv. }\end{array}$ & $\begin{array}{c}\text { Basis Set } B \\
\text { Unsolv. }\end{array}$ & Unsolv. $^{a}$ & Propane & THF $^{c}$ \\
\hline $1988(0.01)$ & $1995(0.11)$ & $1840(0.39)$ & $1859(0.34)$ & $1847(0.27)$ & $1838(0.19)$ \\
$2007(0.08)$ & $2002(0.02)$ & $1977(0.01)$ & $1984(0.01)$ & $1980(0.02)$ & $1975(0.01)$ \\
$2015(0.03)$ & & $1989(0.07)$ & $1990(0.08)$ & $1988(0.14)$ & $1979(0.25)$ \\
$2024(0.61)$ & $2015(0.60)$ & $1997(0.19)$ & $1997(0.16)$ & $1992(0.18)$ & $1983(0.10)$ \\
$2047(1.00)$ & $2038(1.00)$ & $2007(0.43)$ & $2009(0.07)$ & $2012(0.04)$ & $2002(0.01)$ \\
& & $2014(0.11)$ & $2017(0.51)$ & $2018(0.14)$ & $2008(0.21)$ \\
& & $2022(1.00)$ & $2024(0.12)$ & $2024(0.24)$ & $2012(0.23)$ \\
& & $2029(0.12)$ & $2031(1.00)$ & $2032(1.00)$ & $2024(1.00)$ \\
& & $2036(0.38)$ & $2042(0.35)$ & $2039(0.55)$ & $2031(0.45)$ \\
& & $2069(0.37)$ & $2048(0.09)$ & $2062(0.00)$ & $2035(0.02)$ \\
& & $2101(0.03)$ & $2076(0.36)$ & $2076(0.24)$ & $2069(0.15)$ \\
& & & $2109(0.04)$ & $2116(0.02)$ & $2100(0.00)$ \\
\hline
\end{tabular}

${ }^{a}$ Unless otherwise specified, basis set A was used for all calculations. ${ }^{b}$ THF solvated complexes are solvated by the oxygen atom of the THF molecule.

Table S3. Calculated vibrational frequencies $\left(\mathrm{cm}^{-1}\right)$ and relative amplitudes for the carbonyl loss complexes $\mathbf{I I}_{\mathbf{a x}}$ and $\mathbf{I I}_{\mathbf{e q}}$.

\begin{tabular}{c|c|c|c|c|c|c|c}
\hline \multicolumn{4}{c|}{ Complex II } & \multicolumn{4}{c}{ Complex II $_{\text {eq }}$} \\
\hline $\begin{array}{c}\text { Basis Set } B \\
\text { Unsolv. }\end{array}$ & Unsol. $^{a}$ & Propane & THF $^{b}$ & $\begin{array}{c}\text { Basis Set } B \\
\text { Unsolv. }\end{array}$ & Unsolv. & Proane & THF $^{b}$ \\
\hline $1862(0.18)$ & $1884(0.18)$ & $1879(0.21)$ & $1886(016)$. & $1940(0.11)$ & $1963(0.06)$ & $1967(031)$. & $1957(0.04)$ \\
$1948(0.09)$ & $1954(0.11)$ & $1957(0.10)$ & $1926(0.14)$ & $1966(0.20)$ & $1981(0.25)$ & $1982(0.18)$ & $1971(0.16)$ \\
$1972(0.11)$ & $1986(0.11)$ & $1984(0.10)$ & $1975(0.07)$ & $1973(0.18)$ & $1986(0.15)$ & $1986(0.15)$ & $1975(0.12)$ \\
$1994(0.16)$ & $2005(0.37)$ & $2003(0.47)$ & $1993(0.22)$ & $1986(0.05)$ & $1999(0.06)$ & $1994(0.10)$ & $1985(0.17)$ \\
$1996(0.39)$ & $2008(0.19)$ & $2004(0.22)$ & $1995(0.24)$ & $1991(0.07)$ & $2004(0.07)$ & $2002(0.04)$ & $1993(0.05)$ \\
$2008(0.14)$ & $2020(0.16)$ & $2019(0.12)$ & $2009(0.12)$ & $2001(0.05)$ & $2013(0.06)$ & $2010(0.05)$ & $2003(0.02)$ \\
$2012(0.14)$ & $2023(0.11)$ & $2023(0.12)$ & $2012(0.07)$ & $2013(0.74)$ & $2021(0.88)$ & $2017(0.88)$ & $2009(0.80)$ \\
$2022(1.00)$ & $2032(1.00)$ & $2032(1.00)$ & $2021(0.39)$ & $2021(0.18)$ & $2030(0.13)$ & $2026(0.23)$ & $2016(0.40)$ \\
$2026(0.41)$ & $2036(0.40)$ & $2034(0.51)$ & $2023(1.00)$ & $2024(1.00)$ & $2035(1.00)$ & $2034(1.00)$ & $2026(1.00)$ \\
$2043(0.86)$ & $2052(0.88)$ & $2050(0.94)$ & $2041(0.79)$ & $2037(0.74)$ & $2047(0.77)$ & $2044(0.73)$ & $2035(0.79)$ \\
$2086(0.07)$ & $2091(0.07)$ & $2090(0.09)$ & $2082(0.10)$ & $2088(0.05)$ & $2094(0.04)$ & $2092(0.07)$ & $2085(0.08)$ \\
\hline
\end{tabular}

${ }^{a}$ Unless otherwise specified, basis set A was used for all calculations. ${ }^{b}$ THF solvated complexes are solvated by the oxygen atom of the THF molecule. 
